\title{
QUATERNION KÄHLER FLAT MANIFOLDS
}

\author{
ISABEL G. DOTTI AND ROBERTO J. MIATELLO
}

\section{INTRODUCTION}

In this paper we give a method to construct quaternion-Kähler compact flat manifolds. Given any Bieberbach group $\Gamma$ with holonomy group $F$ and translation lattice $\Lambda$ and $\phi: F \rightarrow \mathbb{R}^{n} / \Lambda$, a 1-cocycle modulo $\Lambda$, we form $\mathrm{d}_{\phi} \Gamma$, a Bieberbach group of dimension $2 n$, having holonomy group $F$ and such that the holonomy group commutes with an invariant orthogonal complex structure $J$ on $\mathbb{R}^{n}$. As a second step, we enlarge $\mathrm{d}_{\phi} \Gamma$ into a Bieberbach group with holonomy group $F \times \mathbb{Z}_{2}$, in such a way that the new element of order 2 in the holonomy group anticommutes with $J$. By applying either of these procedures twice, we obtain families of hyperkähler flat manifolds and quaternion Kähler flat manifolds.

In order for the second construction to work, we need a normality condition and a result in [6], therefore we will need to restrict ourselves to certain Bieberbach groups with holonomy group $F \simeq \mathbb{Z}_{2}^{k}$. However, the family of such Bieberbach groups is still very large (see Section 2). Our main result is Theorem 3.5 (see also Corollary 3.6) which will be used in Section 4 to give many families of quaternion Kähler manifolds of dimensions $n \geq 8$, which admit no Kähler structure (see Example 4.1) or no hyperkähler structure (see Example 4.2). This will follow from the explicit calculation of the Betti numbers of the manifolds involved.

\section{Certain Bieberbach groups with holonomy group $\mathbb{Z}_{2}^{k}$}

A Bieberbach group $\Gamma$ is a crystallographic group (i.e. a discrete cocompact subgroup of $\mathrm{I}\left(\mathbb{R}^{n}\right)$ ) which is torsion-free. Hence the action of $\Gamma$ on $\mathbb{R}^{n}$ is without fixed points and the quotient $M_{\Gamma}:=\Gamma \backslash \mathbb{R}^{n}$ is a compact flat Riemannian manifold with fundamental group $\Gamma$. If $v \in \mathbb{R}^{n}$, let $L_{v}$ denote translation by $v$. By Bieberbach's first theorem, if $\Gamma$ is a crystallographic group then $\Lambda=\left\{v: L_{v} \in \Gamma\right\}$ is a lattice in $\mathbb{R}^{n}$. The translation lattice $L_{\Lambda}=\left\{L_{v}: v \in \Lambda\right\}$ is a normal and maximal abelian subgroup of $\Gamma$ and the quotient $F:=L_{\Lambda} \backslash \Gamma$ is a finite group which represents the linear holonomy group of the flat Riemannian manifold $M_{\Gamma}$ and is called the holonomy group of $\Gamma$. We will usually write $\Lambda$ in place of $L_{\Lambda}$ throughout this paper.

Any element $\gamma \in \mathrm{I}\left(\mathbb{R}^{n}\right)$ decomposes uniquely $\gamma=B L_{b}$, with $B \in \mathrm{O}(n)$ and $b \in \mathbb{R}^{n}$ and the lattice $\Lambda$ is $B$-stable for each $B L_{b} \in \Gamma$. The restriction

Dotti and Miatello: Partially supported by Conicet, Conicor and SecytUNC. 
to $\Gamma$ of the canonical projection from $\mathrm{I}\left(\mathbb{R}^{n}\right)$ to $\mathrm{O}(n)$, mapping $B L_{b}$ to $B$, has kernel $\Lambda$ and the image is a finite subgroup of $\mathrm{O}(n)$, called the point group of $\Gamma$. We shall often identify the holonomy group $F$ with the point group of $\Gamma$. The action of $F$ on $\Lambda$ defines an integral representation of $F$, usually called the holonomy representation.

In this paper, for technical reasons, we will be working only with Bieberbach groups with holonomy group $\mathbb{Z}_{2}^{k}$. In Section 3 we will make use of the following result from [6], Proposition 2.1 (see also [9], Proposition 1.1).

Proposition 2.1. Assume that $\Gamma=\left\langle\gamma_{1}, \ldots, \gamma_{k}, \Lambda\right\rangle$ is a subgroup of Aff $\left(\mathbb{R}^{n}\right)$, with $\gamma_{i}=B_{i} L_{b_{i}}, b_{i} \in \mathbb{R}^{n}, B_{i} \in G l(n, \mathbb{R})$ such that $\left\langle B_{1}, \ldots, B_{r}\right\rangle \simeq \mathbb{Z}_{2}^{k}$ and $\Lambda$ is a lattice in $\mathbb{R}^{n}$ stable by the $B_{i}$ 's. Then $\Gamma$ is torsion-free with translation lattice $\Lambda$ if and only if the following two conditions hold:

(i) For each pair $i, j, 1 \leq i, j \leq k, \quad\left(B_{i}-I d\right) b_{j}-\left(B_{j}-I d\right) b_{i} \in \Lambda$.

(ii) For each $I=\left(i_{1}, \ldots, i_{s}\right)$ with $1 \leq i_{1} \leq i_{2} \leq \cdots \leq i_{s} \leq k$, let $B_{i_{1}} L_{b_{i_{1}}} \ldots B_{i_{s}} L_{b_{i_{s}}}=B_{I} L_{b(I)} \in \Gamma$, with $B_{I}:=\bar{B}_{i_{1}} \ldots B_{i_{s}}$ and $b(I)=$ $B_{i_{s}} \ldots B_{i_{2}} b_{i_{1}}+B_{i_{s}} \ldots B_{i_{3}} b_{i_{2}}+\cdots+B_{i_{s}} b_{i_{s-1}}+b_{i_{s}}$. Then

$$
\left(B_{I}+I d\right) b(I) \in \Lambda \backslash\left(B_{I}+I d\right) \Lambda \text {. }
$$

Finally, if $\Gamma$ satisfies conditions (i) and (ii), then $\Gamma$ is isomorphic to a Bieberbach group with holonomy group $F \simeq \mathbb{Z}_{2}^{k}$.

Remark 2.2. We note that the statement in [6] asserts only the sufficiency of conditions (i), (ii) for $\Gamma$ to be torsion-free with translation lattice $\Lambda$, but the converse can be proved, essentially, by reversing the argument given there. Also, although the result in [6] is stated for subgroups of $I\left(\mathbb{R}^{n}\right)$, its proof is valid under the more general conditions above.

We will use a family of Bieberbach groups, introduced in [12] for $F \simeq \mathbb{Z}_{2}^{2}$, that will play a main role in the construction of quaternion flat manifolds in the next section.

Definition 2.3. Let $\Gamma$ be a Bieberbach group with holonomy group $F \simeq \mathbb{Z}_{2}^{k}$ and translation lattice $\Lambda$. Then $\Gamma$ is in class $\mathcal{F}$ if $b \in \frac{1}{2} \Lambda$, for any $B L_{b} \in \Gamma$, and there is a decomposition of $\Lambda: \Lambda=\sum_{j=1}^{r} \Lambda_{j} \oplus \sum_{i=1}^{t} \Lambda_{r+i}$, where $\Lambda_{j}=$ $\mathbb{Z} e_{2 j-1} \oplus \mathbb{Z} e_{2 j}(1 \leq j \leq r), \Lambda_{r+i}=\mathbb{Z} e_{2 r+i}($ for $1 \leq i \leq t)$ are $F$-stable and the holonomy action is given as follows.

For each $B L_{b} \in \Gamma$ and $1 \leq j \leq r, B_{\mid \Lambda_{j}}$ is either $\pm I d$, or the map $\pm V$, where $V$ exchanges $e_{2 j-1}$ and $e_{2 j}$ and, if $1 \leq i \leq t$, then $B e_{2 r+i}= \pm e_{2 r+i}$. Furthermore, each $\Lambda_{j}$ is $F$-indecomposable, that is, for each $j$ there is some $B \in F$ which acts by $\pm V$ on $\Lambda_{j}$.

Remark 2.4. We note that if $\gamma=B L_{b} \in \Gamma$ with $B \in \mathrm{O}(n), b \in \mathbb{R}^{n}$, we may write $b=b^{+}+b^{-}$where $B b^{ \pm}= \pm b^{ \pm}$and since $F \simeq \mathbb{Z}_{2}^{k}$, then $\gamma^{2}=L_{(B+I d) b}=L_{2 b^{+}}$, hence $2 b^{+} \in \Lambda$. However, it may be the case that $2 b^{-} \notin \Lambda$, so we need the assumption that $2 b \in \Lambda$. It can be shown that if the 
holonomy representation is diagonal (i.e. $r=0$ ) then $\Gamma$ can be conjugated in $\mathrm{I}\left(\mathbb{R}^{n}\right)$ to a group $\Gamma^{\prime}$ such that $2 b \in \Lambda$ for each $\gamma$, so in this case this assumption is not necessary (see [9], Section 1). It follows from the known classification (see [1]) that it is also not needed in low dimensions $n \leq 4$.

Although Definition 2.3 may seem rather restrictive, we shall next list many known examples of Bieberbach groups satisfying those conditions, showing that the family $\mathcal{F}$ is actually very large.

Example 2.5. We first use the known classification in low dimensions to describe the Bieberbach groups with holonomy group $\mathbb{Z}_{2}^{k}$ for $n \leq 4$. We refer to [1], p. 408, for a full list of fixed-point-free crystallographic groups in dimensions $\leq 4$.

If $n=2$, the only non-abelian Bieberbach group is, up to conjugation by $\Psi$ in $\operatorname{Aff}\left(\mathbb{R}^{n}\right)$, the Klein bottle group: $\Gamma=\left\langle B L_{\frac{e_{1}}{2}}, \Lambda\right\rangle$ where $B e_{1}=e_{1}, B e_{2}=$ $-e_{2}$. Thus $F \simeq \mathbb{Z}_{2}$ and $\mathrm{r}=0$.

If $n=3$, there are 3 Bieberbach groups with $F \simeq \mathbb{Z}_{2}$, two of them with diagonal holonomy (see [14] or [1]). Namely, $\Gamma_{1}=\left\langle B_{1} L \frac{e_{1}}{2}, \Lambda\right\rangle, \Gamma_{2}=$

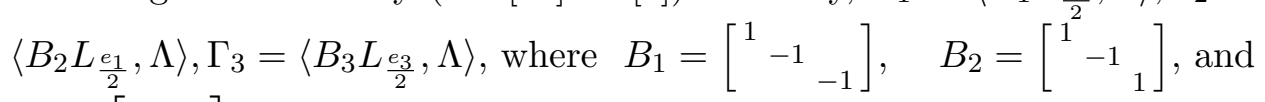
$B_{3}=\left[\begin{array}{ll}V & \\ & 1\end{array}\right]$, where $V$ interchanges $e_{1}$ and $e_{2}$. Also, there are 3 Bieberbach groups with holonomy group $\mathbb{Z}_{2}^{2}$, all of them with diagonal holonomy representation, namely: $\Gamma_{1}=\left\langle B_{1} L_{\frac{e_{1}}{2}}, B_{2} L_{\frac{e_{3}}{2}}, \Lambda\right\rangle, \Gamma_{2}=\left\langle B_{1} L_{\frac{e_{1}}{2}}, B_{2} L_{\frac{e_{2}+e_{3}}{2}}, \Lambda\right\rangle$,

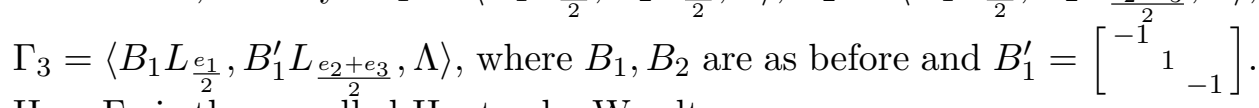
Here $\Gamma_{3}$ is the so called Hantzsche-Wendt group.

If $n=4$ there are 5 groups with $F=\mathbb{Z}_{2}$ (3 of them with $r=0$ and 2 with $r=1$ ). They correspond to families II, III in [1], p 408. Also, there are 26 groups with $F \simeq \mathbb{Z}_{2}^{2}, 21$ of them in $\mathcal{F}$, where 8 are of diagonal type $(r=0)$ and 13 have $r=1$. There are five groups which have a more complicated holonomy representation and are not in class $\mathcal{F}$. These are denoted by IV/04/01/04/005 (p.83), IV/04/01/06/004 (p.84), V/05/01/06/006 (p.90), $\mathrm{V} / 05 / 01 / 07 / 004$ (p.90), $\mathrm{V} / 05 / 01 / 10 / 004$ (p.90) in [1]. We note that, out of the 26 groups with holonomy group $\mathbb{Z}_{2}^{2}, 17$ belong to family IV and 9 to family $\mathrm{V}$ of the list in [1], p 408. Finally, still for $n=4$, there are 12 groups with $F \simeq \mathbb{Z}_{2}^{3}$, all of them in $\mathcal{F}$, with diagonal holonomy representation and all non-orientable (they all belong to family $\mathrm{V}$ in [1], p.408).

Example 2.6. The groups studied in [6], Section 3, have $F=\mathbb{Z}_{2}^{k}$, and belong to family $\mathcal{F}$, for all $n \geq 5,2 \leq k \leq n-3$. Also, in the case when $F \simeq \mathbb{Z}_{2}^{2}$, a subfamily of $\mathcal{F}$ is considered in [11] (see also [5]) and it is shown that its cardinality grows polynomially with $n$. On the other hand, the Hantzsche-Wendt groups in [8] have $F \simeq \mathbb{Z}_{2}^{n-1}$ (n odd) they are of diagonal type, and their number $h(n)$ grows exponentially with $\mathrm{n}$. 
Example 2.7. We now give an example to show that for each $n, r, t$ with $n=2 r+t$, there exists a Bieberbach group $\Gamma_{r, t}$ belonging to family $\mathcal{F}$, with holonomy group $F \simeq \mathbb{Z}_{2}^{r+t-1}$ and where $r, t$ play the role of $r, t$ in Definition 2.3. We let $\Lambda$ be the canonical lattice, decomposed as in Definition 2.3.

For $1 \leq h \leq r$ let $B_{h} \in \mathrm{O}(n)$ be such that $B_{h \mid \Lambda_{j}}=V^{\delta_{h, j}}$ and $B_{h} e_{2 r+i}=$ $e_{2 r+i}$, for $1 \leq j \leq r, 1 \leq i \leq t$. Let $B_{k}^{\prime} \in \mathrm{O}(n)$ be such that $B_{k}^{\prime} e_{i}=$ $(-1)^{\delta_{2 r+k, i}} e_{i}$, for $1 \leq k \leq t-1,1 \leq i \leq n$. Choose $b_{1}=\frac{e_{3}+e_{4}}{2}, \ldots, b_{r-1}=$ $\frac{e_{2 r-1}+e_{2 r}}{2}, b_{r}=\frac{e_{2 r+1}}{2}, b_{1}^{\prime}=\frac{e_{2 r+2}}{2}, b_{2}^{\prime}=\frac{e_{2 r+3}}{2}, \ldots, b_{t-1}^{\prime}=\frac{e_{n}}{2}$.

Set $\Gamma_{r, t}=\left\langle B_{h} L_{b_{h}}, B_{k}^{\prime} L_{b_{k}^{\prime}}, \Lambda: 1 \leq h \leq r, 1 \leq k \leq t-1\right\rangle$.

It is not hard to verify that $\Gamma_{r, t}$ satisfies conditions (i), (ii) of Proposition 2.1 and defines a torsion free group with translation lattice $\Lambda$ which belongs to family $\mathcal{F}$.

Remark 2.8. In the previous examples we listed Bieberbach groups with holonomy group $\mathbb{Z}_{2}^{k}$ which are in class $\mathcal{F}$, except only for the 5 groups listed in Example 2.5 for $n=4$, which have holonomy group $\mathbb{Z}_{2}^{2}$. On the other hand, Nazarova has shown that $\mathbb{Z}_{2}^{2}$ has indecomposable integral representations with arbitrarily large rank ([10]). Using these representations one can obtain Bieberbach groups with holonomy group $\mathbb{Z}_{2}^{2}$ for any $n \geq 5$, which are not in the class $\mathcal{F}$ (see also [12], where all Bieberbach groups with holonomy group $\mathbb{Z}_{2}^{2}$ and $n=5$ are classified).

\section{Construction of quaternion Kähler flat manifolds.}

A Riemannian manifold is quaternion Kähler if its holonomy group is contained in $S p(n) S p(1)$. It is known that quaternion Kähler manifolds are Einstein, so the scalar curvature $s$ splits these manifolds according to whether $s>0, s=0$ or $s<0$. Ricci flat quaternion Kähler manifolds include hyperkähler manifolds, that is, those with full holonomy group contained in $S p(n)$. Such a manifold can be characterized by the existence of a pair of integrable anticommuting complex structures, compatible with respect to the Riemannian metric, and parallel with respect to the Levi-Civita connection.

The simplest model of hyperkähler manifolds (and in particular, of quaternion Kähler manifolds) is provided by $\mathbb{R}^{4 n}$ with the standard flat metric and a pair $J, K$ of orthogonal anticommuting complex structures. This hyperkähler structure descends to the $4 n$-torus $T_{\Lambda}:=\Lambda \backslash \mathbb{R}^{4 n}$, for any lattice $\Lambda$ in $\mathbb{R}^{4 n}$. If $M_{\Gamma}=\Gamma \backslash \mathbb{R}^{4 n}$ is a compact flat manifold such that the holonomy action of $F=\Lambda \backslash \Gamma$ centralizes (resp. normalizes) the algebra generated by $J, K$, then $M_{\Gamma}$ inherits a hyperkähler (resp. quaternion Kähler) structure.

In [3] we introduced a "doubling" procedure for Bieberbach groups which allows to produce many flat hyperkähler (even Clifford Kähler) manifolds. In particular, we showed that any finite group is the holonomy group of a hyperkähler flat manifold. The main goal of this paper is to give a variant of this construction which produces quaternion Kähler manifolds which are 
generically not Kähler. The construction will apply to a large family of Bieberbach groups, including those in family $\mathcal{F}$ (see Section 2). A similar construction should work for Bieberbach groups with more general holonomy groups (using for instance Proposition 1.1 in [MR2]). However, the family to be considered will be sufficiently large to give many rather simple examples of quaternion Kähler flat manifolds which admit no Kähler structure.

Let $\Gamma$ be a Bieberbach group with holonomy group $F$ and translation lattice $\Lambda \subset \mathbb{R}^{n}$. Let $\phi: F \rightarrow \mathbb{R}^{n}$ be a 1-cocycle modulo $\Lambda$, that is, $\phi$ satisfies $\phi\left(B_{1} B_{2}\right)=B_{2}^{-1} \phi\left(B_{1}\right)+\phi\left(B_{2}\right)$, modulo $\Lambda$, for each $B_{1}, B_{2} \in F$. Then $\phi$ defines a cohomology class in $H^{1}\left(F ; \mathbb{R}^{n} / \Lambda\right) \simeq H^{2}(F ; \Lambda)$ and one may associate to $\phi$ a crystallographic group with holonomy group $F$ and translation lattice $\Lambda$. Furthermore, this group is torsion-free if and only if the class of $\phi$ is a special class (see [4]).

Definition 3.1. Let $\Gamma$ be a Bieberbach group with holonomy group $F$ and translation lattice $\Lambda \subset \mathbb{R}^{n}$. Let $\phi: F \rightarrow \mathbb{R}^{n}$ be any 1-cocycle modulo $\Lambda$. We let $\mathrm{d}_{\phi} \Gamma$ be the subgroup of $\mathrm{I}\left(\mathbb{R}^{2 n}\right)$ generated by elements of the form $\left[\begin{array}{ll}B & 0 \\ 0 & B\end{array}\right] L_{(\phi(B), b)}$ and $L_{(\lambda, \mu)}$, for $\gamma=B L_{b} \in \Gamma$ and $(\lambda, \mu) \in \Lambda \oplus \Lambda$.

Proposition 3.2. (compare with [3], Theorem 3.1) Let $\Gamma, \phi$ and $d_{\phi} \Gamma$ be as in Definition 3.1. Then

(i) $d_{\phi} \Gamma$ is a Bieberbach group with holonomy group $F$, translation lattice $\Lambda \oplus \Lambda$ and $d_{\phi} \Gamma \backslash \mathbb{R}^{2 n}$ is a Kähler compact flat manifold.

(ii) If $\Gamma \backslash \mathbb{R}^{n}$ has a locally invariant Kähler structure, then $d_{\phi} \Gamma \backslash \mathbb{R}^{2 n}$ is hyperkähler. In particular, if $\phi^{\prime}: F \rightarrow \mathbb{R}^{2 n}$ is any 1-cocycle modulo $\Lambda \oplus \Lambda$, then $d_{\phi^{\prime}} d_{\phi} \Gamma \backslash \mathbb{R}^{4 n}$ is hyperkähler. Any finite group is the holonomy group of a hyperkähler compact flat manifold.

Proof. Given any two elements $\delta_{1}, \delta_{2} \in \mathrm{d}_{\phi} \Gamma$ with $\delta_{1}=\left[\begin{array}{cc}B_{1} & 0 \\ 0 & B_{1}\end{array}\right] L_{\left(\phi\left(B_{1}\right), b_{1}\right)}$, $\delta_{2}=\left[\begin{array}{cc}B_{2} & 0 \\ 0 & B_{2}\end{array}\right] L_{\left(\phi\left(B_{2}\right), b_{2}\right)}$, we have $\delta_{1} \delta_{2}=\left[\begin{array}{cc}B_{1} B_{2} & 0 \\ 0 & B_{1} B_{2}\end{array}\right] L_{\left(\phi\left(B_{1} B_{2}\right), b\left(B_{1} B_{2}\right)\right)} L_{\left(\mu, \mu^{\prime}\right)}$ where $b\left(B_{1} B_{2}\right)=B_{2}^{-1} b_{1}+b_{2}$ and $\mu, \mu^{\prime} \in \Lambda$.

This implies, since $\Lambda$ is $B$-stable for each $B L_{b} \in \Gamma$, that a generic element in $\mathrm{d}_{\phi} \Gamma$ can be written $\delta=\left[\begin{array}{ll}B & 0 \\ 0 & B\end{array}\right] L_{(\phi(B)+\lambda, b+\mu)}$, for some $B L_{b} \in \Gamma, \lambda, \mu \in \Lambda$. Furthermore we have a homomorphism $\psi: \mathrm{d}_{\phi} \Gamma \rightarrow F$ defined by $\psi(\delta)=B$, having kernel $\Lambda \oplus \Lambda$. It follows that $\mathrm{d}_{\phi} \Gamma$ is a crystallographic subgroup of $\mathrm{I}\left(\mathbb{R}^{2 n}\right)$ with holonomy group $F$ and translation lattice $\Lambda \oplus \Lambda$. Furthermore there is an exact sequence of groups

$$
1 \rightarrow \Lambda \rightarrow \mathrm{d}_{\phi} \Gamma \rightarrow \Gamma \rightarrow 1
$$

where the second map is given by $\delta \rightarrow \gamma=B L_{b} L_{\mu}$, which has kernel $\Lambda \oplus\{0\}$, where $\delta=\left[\begin{array}{cc}B & 0 \\ 0 & B\end{array}\right] L_{(\phi(B)+\lambda, b+\mu)}$, as above.

This implies that $\mathrm{d}_{\phi} \Gamma$ is torsion-free, since $\Gamma$ and $\Lambda$ are torsion-free.

Furthermore, the $\mathrm{O}(2 n)$-component of any element in $\mathrm{d}_{\phi} \Gamma$ commutes with the complex structure $J_{2 n}$ in $\mathbb{R}^{2 n}$ given by $J_{2 n}\left(v_{1}, v_{2}\right)=\left(v_{2},-v_{1}\right)$, thus $\mathrm{d}_{\phi} \Gamma \backslash \mathbb{R}^{2 n}$ is a Kähler compact flat manifold. If $\Gamma \backslash \mathbb{R}^{n}$ is already Kähler, that 
is, if the holonomy action of $F$ commutes with $J_{n}^{\prime}$, a translation invariant complex structure on $\mathbb{R}^{n}$, we put $J_{2 n}^{\prime}=\left[\begin{array}{ll}J_{n}^{\prime} & \\ & -J_{n}^{\prime}\end{array}\right]$, getting a second complex structure which anticommutes with $J_{2 n}$, hence we have a hyperkähler structure on $T^{2 n}$. Since the holonomy action of $\mathrm{d}_{\phi} \Gamma$ commutes with both $J_{2 n}$ and $J_{2 n}^{\prime}$ it follows that $\mathrm{d}_{\phi} \Gamma \backslash \mathbb{R}^{2 n}$ is hyperkähler. This says in particular, that for any Bieberbach group $\Gamma$, if $\phi^{\prime}$ is as in (ii) in the statement, then $\mathrm{d}_{\phi^{\prime}} \mathrm{d}_{\phi} \Gamma \backslash \mathbb{R}^{4 n}$ is hyperkähler. Since by a wellknown theorem of AuslanderKuranishi (see [14], for instance) any finite group is the holonomy group of a compact flat manifold, it follows that any finite group is the holonomy group of a hyperkähler compact flat manifold.

Remark 3.3. In this paper we shall work mostly with the choice $\phi=0$ and we shall then write $\mathrm{d}_{0} \Gamma$. Other natural choice is to let $\phi$ be the 1-cocycle associated to $\Gamma$, as in [3]; we denote $\mathrm{d}_{\phi} \Gamma$ by $\mathrm{d} \Gamma$ in this case.

It is clear that the procedure in (ii) of Proposition 3.2 can be iterated. If we assume that $\phi=0$, for simplicity, and we set $\mathrm{d}_{0}^{m} \Gamma=\mathrm{d}_{0} \mathrm{~d}_{0}^{m-1} \Gamma$, we get that $\mathrm{d}_{0}^{m} \Gamma$ is a Bieberbach subgroup of $\mathrm{I}\left(\mathbb{R}^{2^{m} n}\right)$ with holonomy group $F$, diagonal holonomy representation and translation lattice $\underbrace{\Lambda \oplus \cdots \oplus \Lambda}_{2^{m}}$. Furthermore the holonomy representation commutes with $m$ anticommuting complex structures on $\mathbb{R}^{2^{m} n}$, hence $\mathrm{d}_{0}^{m} \Gamma \backslash \mathbb{R}^{2^{m} n}$ has a Clifford structure of order $m$ (compare [3], 3.1).

We wish to enlarge $\mathrm{d}_{\phi} \Gamma$ into a Bieberbach group $\mathrm{d}_{q, \phi} \Gamma$ in such a way that some element in the holonomy group of $\mathrm{d}_{q, \phi} \Gamma$ anticommutes with the complex structure $J_{2 n}$ in $\mathbb{R}^{2 n}$. Once this is done, then by repeating the procedure twice, we shall get a Bieberbach group such that any element in the holonomy group will either commute or anticommute with each one of a pair of anticommuting complex structures, hence the quotient manifold will be a quaternion Kähler flat manifold which in general, will not be Kähler. In Theorem 3.5 we will show that this can be done for a class of Bieberbach groups with holonomy group $\mathbb{Z}_{2}^{k}$ which includes any group $\Gamma \in \mathcal{F}$ (Section 2).

Definition 3.4. Let $\Gamma$ be a Bieberbach group with holonomy group $F \simeq \mathbb{Z}_{2}^{k}$, with translation lattice $\Lambda$ and such that $b \in \frac{1}{2} \Lambda$ for any $\gamma=B L_{b} \in \Gamma$. Let

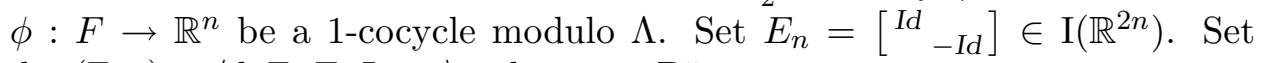
$\mathrm{d}_{q, \phi}(\Gamma, v)=\left\langle\mathrm{d}_{\phi} \Gamma, E_{n} L_{(v, 0)}\right\rangle$, where $v \in \mathbb{R}^{n}$.

As we shall see, under rather general conditions, $\mathrm{d}_{q, \phi}(\Gamma, v)$ contains $\mathrm{d}_{\phi} \Gamma$ as a normal subgroup of index 2 , hence if $v \in \mathbb{R}^{n}$ can be chosen so that $\mathrm{d}_{q, \phi}(\Gamma, v)$ is torsion free, $M_{\mathrm{d}_{q, \phi}(\Gamma, v)}$ will be a compact flat manifold with holonomy group $F \times \mathbb{Z}_{2}$ having as a double cover the Kähler manifold $M_{\mathrm{d}_{\phi} \Gamma}$ (see Definition 3.1). Furthermore $F$ commutes with $J$, but $E_{n}$ only anticommutes with $J$. If we use this construction twice we will get a Bieberbach group $\mathrm{d}_{q}^{2}(\Gamma, v, u):=\mathrm{d}_{q, \phi^{\prime}}\left(\mathrm{d}_{q, \phi}(\Gamma, v), u\right) \subset \mathrm{I}\left(\mathbb{R}^{4 n}\right)$ such that the holonomy group 
normalizes two anticommuting complex structures, $J_{1}, J_{2}$, on $\mathbb{R}^{4 n}$, hence $\mathrm{d}_{q}^{2}(\Gamma, v, u) \backslash \mathbb{R}^{4 n}$ will be a quaternion Kähler manifold. Thus, our main goal will be to give conditions on $v \in \mathbb{R}^{n}$ that ensure that $\mathrm{d}_{q, \phi}(\Gamma, v)$ is torsion free. We also note that if $n$ is even, $M_{\mathrm{d}_{q, \phi}(\Gamma, v)}$ will always be orientable.

Theorem 3.5. Let $\Gamma, \phi$ be as in Definition 3.4. Then

(i) If $v \in \mathbb{R}^{n}$ is such that $2 v \in \Lambda$ and satisfies

$$
(B-I d) v \in \Lambda \text { for each } \gamma=B L_{b} \in \Gamma,
$$

then $d_{q, \phi} \Gamma$ is a crystallographic group with translation lattice $\Lambda \oplus \Lambda$ and holonomy group $\mathbb{Z}_{2}^{k+1}$. Furthermore, $d_{q, \phi} \Gamma$ is torsion-free if and only if $v \notin \Lambda$ and for each $\gamma=B L_{b} \in \Gamma$ we have:

$(B+I d)(\phi(B)+v) \in \Lambda \backslash(B+I d) \Lambda$, or $(B-I d) b \notin(B-I d) \Lambda$.

(ii) If every element in the holonomy group $F$ commutes or anticommutes with a translation invariant complex structure and $v$ satisfies the conditions in (i), then $d_{q, \phi}(\Gamma, v) \backslash \mathbb{R}^{2 n}$ is quaternion Kähler.

(iii) If $v$ satisfies the conditions in (i) we have that $\beta_{1}\left(d_{q, \phi}(\Gamma, v) \backslash \mathbb{R}^{2 n}\right)=$ $\beta_{1}\left(\Gamma \backslash \mathbb{R}^{n}\right)$ and $\beta_{2}\left(d_{q, \phi}(\Gamma, v) \backslash \mathbb{R}^{2 n}\right)=2 \beta_{2}\left(d_{q, \phi}(\Gamma, v) \backslash \mathbb{R}^{2 n}\right)$. Hence, if $\beta_{1}\left(\Gamma \backslash \mathbb{R}^{n}\right)$ is odd, or if $\beta_{2}\left(\Gamma \backslash \mathbb{R}^{n}\right)=0$ and if $F$ satisfies the condition in (ii), then $d_{q, \phi}(\Gamma, v) \backslash \mathbb{R}^{2 n}$ is quaternion Kähler and not Kähler.

(iv) Assume $\phi=0$ and $\Gamma \in \mathcal{F}$ (see Definition 2.1). Then the vector $v=\frac{1}{2} \sum_{i=1}^{n} e_{i}$ satisfies the conditions in $(i)$, hence $d_{q, 0}(\Gamma, v)$ is a Bieberbach group. Furthermore, $d_{q, 0}(\Gamma, v) \in \mathcal{F}$.

Proof. We first show that $\mathrm{d}_{\phi} \Gamma$ is a normal subgroup of index 2 of $\mathrm{d}_{q, \phi}(\Gamma, v)$. We note that for any $\gamma=B L_{b} \in \Gamma$, we have:

$$
E_{n} L_{(v, 0)}\left[\begin{array}{ll}
B & \\
& B
\end{array}\right] L_{(\phi(B), b)}\left(E_{n} L_{(v, 0)}\right)^{-1}=\left[\begin{array}{ll}
B & \\
& B
\end{array}\right] L_{(\phi(B), b)} L_{\left(\mu, \mu^{\prime}\right)}
$$

with $\mu=(B-I d) v, \mu^{\prime}=-2 b$. Now $\mu, \mu^{\prime} \in \Lambda$ by the choice of $v$ and $\Gamma$, hence $\mathrm{d}_{\phi} \Gamma$ is a normal subgroup of $\mathrm{d}_{q, \phi}(\Gamma, v)$. Furthermore, since $2 v \in \Lambda$, any element in $\mathrm{d}_{q, \phi}(\Gamma, v)$ can be written uniquely in one of the following forms:

$$
\left[\begin{array}{ll}
B & \\
& B
\end{array}\right] L_{\left(\phi(B)+\lambda, b+\lambda^{\prime}\right)},\left[\begin{array}{ll}
B & \\
& -B
\end{array}\right] L_{\left(\phi(B)+v+\lambda,-b+\lambda^{\prime}\right)}
$$

with $\lambda, \lambda^{\prime} \in \Lambda, \gamma=B L_{b} \in \Gamma$. This implies that if we map the elements of the second (resp. first) type to -1 (resp. 1) we get an epimorphism $\pi: \mathrm{d}_{q, \phi}(\Gamma, v) \rightarrow \mathbb{Z}_{2}$ with kernel $\mathrm{d}_{\phi} \Gamma$. Thus, there is an exact sequence

$$
1 \rightarrow \mathrm{d}_{\phi} \Gamma \rightarrow \mathrm{d}_{q, \phi}(\Gamma, v) \rightarrow \mathbb{Z}_{2} \rightarrow 1,
$$

hence $\mathrm{d}_{q, \phi}(\Gamma, v)$ is a crystallographic group.

The general expression of the elements of $\mathrm{d}_{q, \phi}(\Gamma, v)$ given in (1) ensures that the translation lattice of $\Gamma$ is $\Lambda \oplus \Lambda$ and the holonomy group is $F \times \mathbb{Z}_{2}$.

We now find conditions on $v, \Gamma$ for $\mathrm{d}_{q, \phi}(\Gamma, v)$ to be torsion-free. Proposition 2.1 (ii) requires that $2 v \in \Lambda \backslash 2 \Lambda$ and secondly (using the general form 
(1) of an element in $\mathrm{d}_{q, \phi}(\Gamma, v)$ which is not in $\left.\mathrm{d}_{\phi} \Gamma\right)$, that for any $B L_{b} \in \Gamma$ one of the following holds:

$$
(B+I d)(\phi(B)+v) \in \Lambda \backslash(B+I d) \Lambda \text { or }(B-I d) b \in \Lambda \backslash(B-I d) \Lambda .
$$

Since we know that $(B+I d) b \in \Lambda(\Gamma$ has translation lattice $\Lambda)$ and $2 b$ lies in $\Lambda$, the previous condition is equivalent to the one asserted in (i).

The proof of (ii) is the same as the one given in the proof of the analogous assertion in Proposition 3.2, (i).

In order to prove (iv), we will need some general properties of the elements of $\Gamma \in \mathcal{F}$. We keep the notation from Section 2 (see Definition 2.3).

(a) For any $B L_{b} \in \Gamma, B_{\mid \Lambda_{j}}= \pm I d$ or $\pm V$, for $1 \leq j \leq r$. Indeed, since for any $j$ there is some element $B^{\prime} L_{b^{\prime}} \in \Gamma$ which acts by $\pm V$ on $\Lambda_{j}$ and $B$ commutes with $B^{\prime}$, then $B_{\mid \Lambda_{j}}$ must commute with $V$, hence the only possibilities are $B_{\mid \Lambda_{j}}= \pm I d$ or $B_{\mid \Lambda_{j}}= \pm V$, since $B^{\prime} \in \mathrm{O}(n)$. Furthermore, $B e_{2 r+i}= \pm e_{2 r+i}$, for $1 \leq i \leq t$.

(b) Since $2 b \in \Lambda$ for any $B L_{b} \in \Gamma$ then, modulo $\Lambda$, we have that $2 b=$ $\sum_{j=1}^{2 r+t} a_{j} e_{j}$, with $a_{j}=0,1$ for all $j$. We claim that $a_{2 j-1}=a_{2 j}$ for $1 \leq j \leq r$, that is:

$$
b=\frac{1}{2} \sum_{j=1}^{r} c_{j}\left(e_{2 j-1}+e_{2 j}\right)+\frac{1}{2} \sum_{i=1}^{t} d_{i} e_{2 r+i}
$$

for some coefficients $c_{j}, d_{i}$ in $\{0,1\}$, for all $j$. We note that this expression implies in particular that $(B \pm I d) b \in \Lambda$.

Let $p_{j}$ denote the projection onto $\Lambda_{j}$ according to the decomposition in Definition 2.2. To show that $b$ can be written as in (2) we note that if $B L_{b} \in \Gamma$ satisfies $B_{\mid \Lambda_{j}}= \pm V$, then, the projection $p_{j}$ of $b$ on $\Lambda_{j}$ must be either 0 , or $\frac{e_{2 j-1}+e_{2 j}}{2}$, modulo $\Lambda$. Indeed, since $(B+I d) p_{j} b \in \Lambda$, one can not have $p_{j} b$ equal to $\frac{e_{2 j-1}}{2}$ or $\frac{e_{2 j}}{2}$.

Now we assume that $j$ is such that $B$ acts on $\Lambda_{j}$ by $\pm I$. Let $B^{\prime} L_{b^{\prime}} \in \Gamma$ be so that $B^{\prime}$ acts on $\Lambda_{j}$ by $\pm V$. We have seen already that $p_{j} b^{\prime}$ is either 0 or $\frac{e_{2 j-1}+e_{2 j}}{2}$. Since $B L_{b} B^{\prime} L_{b^{\prime}}=B B^{\prime} L_{B^{\prime} b+b^{\prime}}$, we have that $\left(B B^{\prime}+I d\right)\left(B^{\prime} b+\right.$ $\left.b^{\prime}\right) \in \Lambda$. We claim that this implies that $p_{j} b$ is either 0 or $\frac{e_{2 j-1}+e_{2 j}}{2}$. Since $\left(B B^{\prime}+I d\right)$ acts on $\Lambda_{j}$ by $\pm V+I d$, then we have that, $\bmod \Lambda$,

$$
p_{j}\left(B B^{\prime}+I d\right)\left(B^{\prime} b+b^{\prime}\right)=\left(B+B^{\prime}\right) p_{j} b+\left(B B^{\prime}+I d\right) p_{j} b^{\prime} \equiv( \pm I+ \pm V) p_{j} b,
$$

since $\left( \pm B^{\prime}+I d\right) p_{j} b^{\prime} \in \Lambda$. It thus follows that $( \pm V+ \pm I d) p_{j} b \in \Lambda$, and this clearly implies that, modulo $\Lambda, p_{j} b$ is either 0 or $\frac{e_{2 j-1}+e_{2 j}}{2}$ as asserted. This concludes the proof of $(b)$.

(c) For any $B L_{b} \in \Gamma, b=b^{+}+b^{-}$where $B b^{ \pm}= \pm b^{ \pm}$; now $\Gamma$ torsionfree implies $b^{+} \neq 0$ (otherwise $\left(B L_{b}\right)^{2}=I d$ ). Furthermore, $b^{+}$must have a nonzero projection onto some $\Lambda_{j}(1 \leq j \leq r)$ where $B$ acts by $I d$, or it must have a nonzero projection onto some space $\mathbb{R} e_{2 r+i}(1 \leq i \leq t)$, where $e_{2 r+i}$ 
is fixed by $B$. To prove this assertion we note that if $p_{j} b:=\frac{e_{2 j-1}+e_{2 j}}{2}$ and $B_{\mid \Lambda_{j}}= \pm V$, then $p_{j}(B+I d) b=\left(e_{2 j-1}+e_{2 j}\right)=(B+I d) e_{2 j-1} \in(B+I d) \Lambda$, if $B$ acts by $V$, or else $p_{j}(B+I d) b=0$, if $B$ acts by $-V$. This says that for this $j, p_{j} b^{+}$does not satisfy condition (ii) in Proposition 2.1. Therefore $b$ must necessarily have some nonzero projection of the form asserted above.

Now we are in a position to prove (iv) in the theorem. If we choose $v=$ $\frac{1}{2} \sum_{i=1}^{2 r+t} e_{j}$, then $2 v \in \Lambda \backslash 2 \Lambda$. Furthermore by (c), $(B+I d)_{\mid \Lambda_{j}}=2 I d$, for some $1 \leq j \leq r$, or else $B+I d$ fixes $e_{2 r+i}$ for some $1 \leq i \leq t$. In the first case we see that $p_{j}(B+I d) v=e_{2 j-1}+e_{2 j}$ which is not in $(B+I d) \Lambda_{j}=\mathbb{Z} 2\left(e_{2 j-1}+e_{2 j}\right)$. In the second case, $p_{2 r+i}(B+I d) v=e_{2 r+i} \notin(B+I d) \mathbb{Z} e_{i}=\mathbb{Z} 2 e_{i}$. Thus, this choice of $v$ satisfies all the conditions in (i) of the theorem, for $\Gamma$ to be torsion-free. Hence (iv) is proved.

Concerning (iii), we note that by [7], the Betti numbers of a compact flat manifold with fundamental group $\Gamma$ are given by $\beta_{j}\left(M_{\Gamma}\right)=\operatorname{dim} \Lambda^{j} \mathbb{R}^{n^{\mathrm{F}}}$, where the $F$-invariants are taken with respect to the rational holonomy action of $F$. We note that the action over $\mathbb{Q}$ is always diagonal, for $\Gamma$ with holonomy group $\mathbb{Z}_{2}^{k}$. Thus $\beta_{1}\left(M_{\Gamma}\right)$ is the dimension of the space of $F$-fixed vectors in $\mathbb{R}^{n}$. In our case, since $E_{n}$ acts by $-I d$ on each $e_{i}$ for $n+1 \leq i \leq 2 n$, it follows that $\beta_{1}\left(M_{d_{\phi, q} \Gamma}\right)=\beta_{1}\left(M_{\Gamma}\right)$. The assertion on $\beta_{2}$ is checked similarly, by observing that the $F \times \mathbb{Z}_{2}$-fixed vectors on $\Lambda^{2} \mathbb{R}^{2 n}$ are obtained by taking the exterior product of two vectors $v, v^{\prime}$ lying both either in $\sum_{1}^{n} \mathbb{R} e_{i}$, or in $\sum_{n+1}^{2 n} \mathbb{R} e_{i}$, and where $v, v^{\prime}$ are such that each element in $F$ acts by multiplication either by 1 , or by -1 , on both of $v, v^{\prime}$. This concludes the proof of the theorem.

Corollary 3.6. In the notation of Theorem 3.5, assume $v \in \mathbb{R}^{n}$ is such that $d_{q, \phi}(\Gamma, v)$ is a Bieberbach group. Let $\phi^{\prime}$ be a cocycle on $F$ modulo $\Lambda \oplus$ $\Lambda$. If $u \in \mathbb{R}^{2 n}$ can be chosen so that $d_{q, \phi, \phi^{\prime}}^{2}(\Gamma, v, u):=d_{q, \phi^{\prime}}\left(d_{q, \phi}(\Gamma, v), u\right)$ is torsion-free, then the quotient of $\mathbb{R}^{4 n}$ by $d_{q, \phi, \phi^{\prime}}^{2}(\Gamma, v, u)$ is a quaternion Kähler manifold. In particular, if $\Gamma$ is a Bieberbach group in $\mathcal{F}$ and we take $\phi=0, v=\sum_{i=1}^{n} e_{i}$ and $u=\sum_{i=2 n+1}^{3 n} e_{i}$, then $d_{q, 0}(\Gamma, v) \in \mathcal{F}$ and $d_{q, 0,0}^{2}(\Gamma, v, u) \backslash \mathbb{R}^{4 n}$ is a quaternion Kähler manifold.

Remark 3.7. As it will be seen in the next section (see Examples 4.1, 4.2) the vector $v$ satisfying the conditions in the theorem is by no means unique, in general.

Remark 3.8. Properties (b) and (c) in the proof of Theorem 3.5 imply that for any $\Gamma \in \mathcal{F}$ with holonomy group $\mathbb{Z}_{2}^{k}$, the relation $k \leq r+t$ must hold. Indeed, by (c), the subgroup $S$ of $\frac{1}{2} \Lambda$ generated by $\left\{b: B \bar{L}_{b} \in \Gamma\right.$, for some $B \in$ $\mathrm{O}(n)\}$ coincides with the subgroup generated by $\left\{b_{i}: 1 \leq i \leq k\right\}$, in the notation of Proposition 2.1. Hence if $F \simeq \mathbb{Z}_{2}^{k}$, then $S$ has rank at most $k$. Now, it is easy to see that since $\Gamma$ is torsion-free, $b_{i_{1}}+\cdots+b_{i_{h}} \neq 0$, $\bmod \Lambda$, hence the rank must be exactly $k$. Furthermore, it follows from (b) in the theorem, that $S$ is contained in the subgroup $S_{r, t}$ generated by 
$\left\{\frac{e_{2 j-1}+e_{2 j}}{2}, \frac{e_{2 r+i}}{2}: 1 \leq j \leq r, 1 \leq i \leq t\right\}$, which has rank $r+t$. We also note that if $r=0$, then $S$ can not have rank $t$, since $F \simeq \mathbb{Z}_{2}^{t}$, would imply $-I d \in F$, hence $\Gamma$ can not be torsion-free. Therefore we must have $k \leq t-1$, if $r=0$. Actually, all examples known to us indicate that any $\Gamma \in \mathcal{F}$ should satisfy the relation $k \leq r+t-1$. If this relation held, it would then follow that the rank of $S$ is at most $r+t-1$. We note that, since the groups $\Gamma_{r, t}$ in Example 2.7 satisfy $k=r+t-1$, a better inequality than $k \leq r+t-1$ can not be obtained.

\section{Quaternion Kähler flat MANifolds of LOW Dimensions}

We will now illustrate the construction and results in the previous section by looking at several particular Bieberbach groups in low dimensions. In almost all cases we will use $\phi=0$ and we will write $\mathrm{d}_{q, 0}^{2}(\Gamma, v, u)$ in place of $\mathrm{d}_{q, 0}\left(\mathrm{~d}_{q, 0}(\Gamma, v), u\right)$. Furthermore it will be convenient, for any $C$ in $\mathrm{O}(n)$, to denote by $C^{\prime} \in \mathrm{O}(2 n)$ the matrix $C^{\prime}=\left[{ }^{C}{ }_{C}\right]$. Also, $C^{\prime \prime} \in \mathrm{O}(4 n)$ will have a similar meaning and $\Lambda_{n}$ will denote the canonical lattice in $\mathbb{R}^{n}$.

Example 4.1. We let first $\Gamma$ be the Klein bottle Bieberbach group, for $n=2$. By applying $\mathrm{d}_{q, 0}$ twice to $\Gamma$, we shall obtain several 8-dimensional compact flat manifolds with holonomy group $\mathbb{Z}_{2}^{3}$ which are quaternion Kähler and not Kähler. This will follow from the explicit computation of the real cohomology.

We take $\Gamma=\left\langle B L_{b}, \Lambda_{2}\right\rangle$, where $B=\left[{ }^{1}{ }_{-1}\right], b=\frac{e_{1}}{2}$. Then $\Gamma \backslash \mathbb{R}^{2}$ is a Klein bottle. If $v=\frac{1}{2}\left(m_{1} e_{1}+m_{2} e_{2}\right), m_{1}, m_{2} \in \mathbb{Z}$, then

$$
\mathrm{d}_{q, 0}(\Gamma, v)=\left\langle B^{\prime} L_{b^{\prime}}, E_{2} L_{(v, 0)}, \Lambda_{4}\right\rangle,
$$

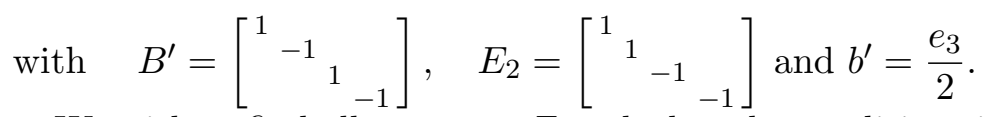

We wish to find all $m_{1}, m_{2} \in \mathbb{Z}$ such that the conditions in (i) of Theorem 3.5 are satisfied, so that $\mathrm{d}_{q, 0}(\Gamma, v)$ is torsion-free.

The first condition in (i) clearly holds for any choice of $v$ since $(B-I d) v=$ $-m_{2} e_{2} \in \Lambda_{2}$. Furthermore, $v \in \frac{1}{2} \Lambda \backslash \Lambda$ if and only if at least one of the $m_{i}$ 's is odd. We also need that $(B+I d) v=m_{1} e_{1} \notin(B+I d) \Lambda_{2}=\mathbb{Z} 2 e_{1}$, hence $m_{1}$ must be odd. Thus, the possible solutions, modulo $\Lambda_{2}$ are $v_{1}=\frac{e_{1}}{2}$ and $v_{2}=\frac{e_{1}+e_{2}}{2}$. By computing the first integral homology groups in both cases, one can show that these solutions lead to flat manifolds non homeomorphic to each other.

We now form $\mathrm{d}_{q, 0}^{2}\left(\Gamma, v_{i}, u\right)$ with $i=1,2$ and $u=\frac{1}{2} \sum_{j=1}^{4} m_{j} e_{j}$, with $m_{j} \in \mathbb{Z}$ to be determined. Again we need that at least one of the $m_{j}^{\prime} \mathrm{s}$ be odd. We now consider the second condition in (i) for each choice of $v$.

We have that

$$
\mathrm{d}_{q, 0}^{2}\left(\Gamma, \frac{e_{1}}{2}, u\right)=\left\langle B^{\prime \prime} L_{\frac{e_{7}}{2}}, E_{2}^{\prime} L_{\frac{e_{5}}{2}}, E_{4} L_{(u, 0)}, \Lambda_{8}\right\rangle
$$




$$
\mathrm{d}_{q, 0}^{2}\left(\Gamma, \frac{e_{1}+e_{2}}{2}, u\right)=\left\langle B^{\prime \prime} L_{\frac{e_{7}}{2}}, E_{2}^{\prime} L_{\frac{e_{5}+e_{6}}{2}}, E_{4} L_{(u, 0)}, \Lambda_{8}\right\rangle
$$

where

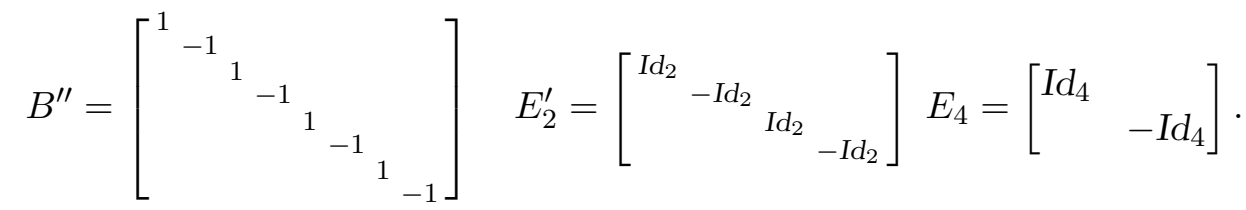

The first condition in (i) is clearly satisfied in both cases, for any choice of $u \in \frac{1}{2} \Lambda$, since the matrices $B^{\prime}, E_{2}$ are diagonal. For the second condition we also need:

$$
\begin{aligned}
\left(B^{\prime}+I d\right) u & \left.=m_{1} e_{1}+m_{3} e_{3} \notin\left(B^{\prime}+I d\right) \Lambda_{4}=\mathbb{Z} 2 e_{1} \oplus \mathbb{Z} 2 e_{3}\right), \\
\left(E_{2}+I d\right) u & \left.=m_{1} e_{1}+m_{2} e_{2} \notin\left(E_{2}+I d\right) \Lambda_{4}=\mathbb{Z} 2 e_{1} \oplus \mathbb{Z} 2 e_{2}\right), \\
\left(B^{\prime} E_{2}+I d\right) u & \left.=m_{1} e_{1}+m_{4} e_{4} \notin\left(B^{\prime} E_{2}+I d\right) \Lambda_{4}=\mathbb{Z} 2 e_{1} \oplus \mathbb{Z} 2 e_{4}\right) .
\end{aligned}
$$

These conditions are satisfied if and only if, either $m_{1}$ is odd, or if each one of $m_{2}, m_{3}$ and $m_{4}$ are odd. This yields the following solutions modulo $\Lambda_{4}$ : either $u=u_{Q}=\frac{e_{1}+e_{Q}}{2}$, where $e_{Q}=\sum_{j \in Q} e_{j}$ and $Q$ runs through all subsets of $\{2,3,4\}$, or $u=u^{\prime}:=\frac{e_{2}+e_{3}+e_{4}}{2}$. We get 9 distinct solutions, the same set for both choices $v=v_{1}, v=v_{2}$. It will be convenient to order the subsets $Q$ as follows: $\emptyset,\{2\},\{3\},\{4\},\{2,3\}\{2,4\}\{3,4\},\{2,3,4\}$ and then to set $u_{j}=u_{Q}$, for $j=1, \ldots, 8$ according to this ordering, letting $u_{9}=u^{\prime}$.

In this way we obtain 18 Bieberbach groups $\Gamma_{i, j}:=\mathrm{d}_{q, 0}^{2}\left(\Gamma, v_{i}, u_{j}\right)$ with $1 \leq i \leq 2,1 \leq j \leq 9$, so that the quotients $\Gamma_{i, j} \backslash \mathbb{R}^{8}$ are quaternion Kähler manifolds. We note that none of these manifolds is Kähler, since for all $i, j, \beta_{1}\left(\Gamma_{i, j} \backslash \mathbb{R}^{8}\right)=\beta_{1}\left(\Gamma \backslash \mathbb{R}^{2}\right)=1$ and $\beta_{2}\left(\Gamma_{i, j} \backslash \mathbb{R}^{8}\right)=2 \beta_{2}\left(\Gamma \backslash \mathbb{R}^{2}\right)=0$, by (iii) in Theorem 3.5. We also note that some of the groups may possibly be isomorphic to each other, however we will see later that many of them are pairwise non isomorphic, by computing $\Gamma_{i, j} /\left[\Gamma_{i, j}, \Gamma_{i, j}\right]$ in each case.

We shall first determine all Betti numbers, by giving generators of $\Lambda^{h} \mathbb{R}^{8^{\mathrm{F}}}$, for $1 \leq h \leq 8$.

It is clear that the space of $F$-invariants in $\mathbb{R}^{8}$ is spanned by $e_{1}$ and furthermore $\Lambda^{2} \mathbb{R}^{8} \mathrm{~F}=0$. If $h=3$, it is easy to see that a basis for the $F$-invariants is given by $e_{3} \wedge e_{5} \wedge e_{7}, e_{2} \wedge e_{3} \wedge e_{4}, e_{3} \wedge e_{6} \wedge e_{8}, e_{2} \wedge e_{5} \wedge e_{6}, e_{2} \wedge$ $e_{7} \wedge e_{8}, e_{4} \wedge e_{6} \wedge e_{7}, e_{4} \wedge e_{5} \wedge e_{8}$, hence $\beta_{3}=\beta_{5}=7$.

By Poincaré duality we have that $\chi\left(\Gamma_{i, j} \backslash \mathbb{R}^{8}\right)=2-2 \beta_{1}+2 \beta_{2}-2 \beta_{3}+\beta_{4}=0$, hence (since $\beta_{1}=1, \beta_{2}=0, \beta_{3}=7$ ) we get $\beta_{4}=2 \beta_{3}=14$. We may check this value by finding a basis for the $F$-invariants in $\Lambda^{4} \mathbb{R}^{8}$. This is given by vectors of the form $e_{i} \wedge e_{j} \wedge e_{k} \wedge e_{l}$, with $\{i, j, k, l\}$ running through the sets $\{1,3,5,7\},\{2,4,6,8\},\{1,2,5,6\},\{3,4,7,8\},\{2,3,5,8\},\{1,2,3,4\}$, $\{5,6,7,8\},\{1,3,6,8\},\{1,2,7,8\},\{2,4,5,7\},\{1,4,6,7\},\{2,3,6,7\},\{2,4,5,7\}$ and $\{1,4,5,8\}$.

Summing up, we get that the Poincaré polynomial of each one of the flat manifolds $\Gamma_{i, j} \backslash \mathbb{R}^{8}$ is $p(t)=1+t+7 t^{3}+14 t^{4}+7 t^{5}+t^{7}+t^{8}$. 
We thus have 2-fold coverings $M_{\mathrm{d}_{0}^{2} \Gamma} \rightarrow M_{\Gamma_{i j}}$, where $M_{\mathrm{d}_{0}^{2} \Gamma}$ is hyperkähler, by Proposition 3.2, and $M_{\Gamma_{i, j}}$ does not admit any Kähler structure, since $\beta_{1}\left(M_{\Gamma_{i, j}}\right)=1$, for all $i, j$.

To conclude this example, we shall show that many of the manifolds $M_{\Gamma_{i j}}$ are non homeomorphic to each other, by computing the first integral homology groups, $H_{1}\left(M_{\Gamma_{i j}}, \mathbb{Z}\right) \simeq \Gamma_{i j} /\left[\Gamma_{i j}, \Gamma_{i j}\right]$.

We first consider the case when $i=1, j \neq 9$. Set $\gamma_{1}=B^{\prime \prime} L_{\frac{e_{7}}{2}}, \gamma_{2}=$ $E_{2}^{\prime} L_{\frac{e_{5}}{2}}, \gamma_{3}=E_{4} L_{\frac{e_{1}+e_{Q}}{2}}$ with $Q \subset\{2,3,4\}$ and $e_{Q}=\sum_{j \in Q} e_{j}$. We have that $\left[\gamma, L_{e_{k}}\right]=L_{2 e_{k}}$, if $\gamma=B L_{b}$ and $B e_{k}=-e_{k}$. Also, we compute that

$$
\left[\gamma_{1}, \gamma_{2}\right]=L_{e 7},\left[\gamma_{1}, \gamma_{3}\right]=L_{e_{7}+\left(B^{\prime \prime}-I d\right) \frac{e_{Q}}{2}},\left[\gamma_{2}, \gamma_{3}\right]=L_{e_{5}+\left(E_{2}^{\prime}-I d\right) \frac{e_{Q}}{2}} .
$$

We note that $e_{Q}^{\prime \prime}:=\left(B^{\prime \prime}-I d\right) \frac{e_{Q}}{2}$ and $e_{Q}^{\prime}:=\left(E_{2}^{\prime}-I d\right) \frac{e_{Q}}{2}$ equal minus the orthogonal projections of $e_{Q}$ onto the spaces $\mathbb{R e}_{2}+\mathbb{R} e_{4}$ and $\mathbb{R e}_{3}+\mathbb{R} e_{4}$, respectively. If $j=9$, then $\gamma_{3}=E_{4} L_{\frac{e_{2}+e_{3}+e_{4}}{2}}$ and the commutation relations are the same as in the case when $j=8$, since $e_{1}$ is fixed by $B^{\prime \prime}, E_{2}^{\prime}$ and $E_{4}$. We therefore have, for $1 \leq j \leq 9$,

$$
\left[\Gamma_{1, j}, \Gamma_{1, j}\right]=\left\langle L_{e_{7}}, L_{e_{7}+e_{Q}^{\prime \prime}}, L_{e_{5}+e_{Q}^{\prime}}, L_{2 e_{j}}: j \neq 1\right\rangle .
$$

Taking into account that $\gamma_{1}^{2}=L_{e_{7}}, \gamma_{2}^{2}=L_{e_{5}}, \gamma_{3}^{2}=L_{e_{1}+e_{Q}}\left(\right.$ resp. $\gamma_{3}^{2}=$ $L_{e_{2}+e_{3}+e_{4}}$ if $\left.j=9\right)$, we determine the groups $\Gamma_{1, j} /\left[\Gamma_{1, j}, \Gamma_{1, j}\right] \simeq H_{1}\left(M_{\Gamma_{1, j}}, \mathbb{Z}\right)$ for each $1 \leq j \leq 9$. These are given in the following table:

\begin{tabular}{|c|c|c|}
\hline \hline$Q$ & {$\left[\Gamma_{1, j}, \Gamma_{1, j}\right]$} & $H_{1}\left(M_{\Gamma_{1, j}}, \mathbb{Z}\right)$ \\
\hline \hline$\emptyset$ & $\left\langle L_{e_{7}}, L_{e_{5}}, L_{2 e_{j}}: j \neq 1\right\rangle$ & $\mathbb{Z} \oplus \mathbb{Z}_{2}^{7}$ \\
\hline$\{2\}$ & $\left\langle L_{e_{7}}, L_{e_{2}}, L_{e_{5}}, L_{2 e_{j}}: j \neq 1\right\rangle$ & $\mathbb{Z} \oplus \mathbb{Z}_{2}^{6}$ \\
\hline$\{3\}$ & $\left\langle L_{e_{7}}, L_{e_{5}+e_{3}}, L_{2 e_{j}}: j \neq 1\right\rangle$ & $\mathbb{Z} \oplus \mathbb{Z}_{4} \oplus \mathbb{Z}_{2}^{6}$ \\
\hline$\{4\}$ & $\left\langle L_{e_{7}}, L_{e_{4}}, L_{e_{5}}, L_{2 e_{j}}: j \neq 1\right\rangle$ & $\mathbb{Z} \oplus \mathbb{Z}_{2}^{6}$ \\
\hline$\{2,3\}$ & $\left\langle L_{e_{7}}, L_{e_{2}}, L_{e_{5}+e_{3}}, L_{2 e_{j}}: j \neq 1\right\rangle$ & $\mathbb{Z} \oplus \mathbb{Z}_{4} \oplus \mathbb{Z}_{2}^{5}$ \\
\hline$\{2,4\}$ & $\left\langle L_{e_{7}}, L_{e_{2}+e_{4}}, L_{e_{5}+e_{4}}, L_{2 e_{j}}: j \neq 1\right\rangle$ & $\mathbb{Z} \oplus \mathbb{Z}_{4} \oplus \mathbb{Z}_{2}^{5}$ \\
\hline$\{3,4\}$ & $\left\langle L_{e_{7}}, L_{e_{4}}, L_{e_{5}+e_{3}}, L_{2 e_{j}}: j \neq 1\right\rangle$ & $\mathbb{Z} \oplus \mathbb{Z}_{4} \oplus \mathbb{Z}_{2}^{5}$ \\
\hline$\{2,3,4\}$ & $\left\langle L_{e_{7}}, L_{e_{2}+e_{4}}, L_{e_{5}+e_{3}+e_{4}}, L_{2 e_{j}}: j \neq 1\right\rangle$ & $\mathbb{Z} \oplus \mathbb{Z}_{4} \oplus \mathbb{Z}_{2}^{5}$ \\
\hline$j=9$ & $\left\langle L_{e_{7}}, L_{e_{2}+e_{4}}, L_{e_{5}+e_{3}+e_{4}}, L_{2 e_{j}}: j \neq 1\right\rangle$ & $\mathbb{Z} \oplus \mathbb{Z}_{4}^{2} \oplus \mathbb{Z}_{2}^{4}$ \\
\hline \hline \multicolumn{3}{|c}{}
\end{tabular}

We thus get 5 different values for $H_{1}\left(M_{\Gamma_{1, j}}, \mathbb{Z}\right)$, namely $\mathbb{Z} \oplus \mathbb{Z}_{2}^{h}$, with $h=6,7, \mathbb{Z} \oplus \mathbb{Z}_{4} \oplus \mathbb{Z}_{2}^{k}$, with $k=5,6$ and $\mathbb{Z} \oplus \mathbb{Z}_{4}^{2} \oplus \mathbb{Z}_{2}^{4}$.

We now consider the case of $\Gamma_{2, j}$. Then $\gamma_{1}=B^{\prime \prime} L_{\frac{e_{7}}{2}}, \gamma_{2}=E_{2}^{\prime} L_{\frac{e_{5}+e_{6}}{2}}, \gamma_{3}=$ $E_{4} L_{\frac{e_{1}+e_{Q}}{2}}$ with $Q \subset\{2,3,4\}$ and $e_{Q}=\sum_{j \in Q} e_{j}$, if $1 \leq j \leq 8$. If $j \stackrel{2}{=} 9$, then $\gamma_{3}=E_{4}^{2} L_{\frac{e_{2}+e_{3}+e_{4}}{2}}$. We have that $\left[\gamma, L_{e_{k}}\right]=L_{2 e_{k}}$, if $\gamma=B L_{b}$ and $B e_{k}=-e_{k}$. Also, we compute that

$$
\left[\gamma_{1}, \gamma_{2}\right]=L_{-e_{7}-e_{6}}, \quad\left[\gamma_{1}, \gamma_{3}\right]=L_{e_{7}+e_{Q}^{\prime \prime}}, \quad\left[\gamma_{2}, \gamma_{3}\right]=L_{e_{5}+e_{6}+e_{Q}^{\prime}}
$$


We have

$$
\left[\Gamma_{2, j}, \Gamma_{2, j}\right]=\left\langle L_{e_{7}+e_{6}}, L_{e_{7}+e_{Q}^{\prime \prime}}, L_{e_{5}+e_{6}+e_{Q}^{\prime}}, L_{2 e_{j}}: j \neq 1\right\rangle,
$$

and furthermore, $\gamma_{1}^{2}=L_{e_{7}}, \gamma_{2}^{2}=L_{e_{5}+e_{6}}, \gamma_{3}^{2}=L_{e_{1}+e_{Q}}, 1 \leq j \leq 8$ and $\gamma_{3}^{2}=L_{e_{2}+e_{3}+e_{4}}$, if $j=9$. We thus get that the groups $\Gamma_{2, j} /\left[\Gamma_{2, j}, \Gamma_{2, j}\right] \simeq$ $H_{1}\left(M_{\Gamma_{2, j}}, \mathbb{Z}\right)$ are as given in the following table:

\begin{tabular}{|c|c|c|}
\hline$Q$ & {$\left[\Gamma_{2, j}, \Gamma_{2, j}\right]$} & $H_{1}\left(M_{\Gamma_{2, j}}, \mathbb{Z}\right)$ \\
\hline$\overline{\varnothing \emptyset}$ & $\left\langle L_{e_{7}+e_{6}}, L_{e_{7}}, L_{e_{5}+e_{6}}, L_{2 e_{j}}: j \neq 1\right\rangle$ & $\mathbb{Z} \oplus \mathbb{Z}_{2}^{7}$ \\
\hline$\{2\}$ & $\left\langle L_{e_{7}+e_{6}}, L_{e_{7}+e_{2}}, L_{e_{5}+e_{6}}, L_{2 e_{j}}: j \neq 1\right\rangle$ & $\mathbb{Z} \oplus \mathbb{Z}_{4} \oplus \mathbb{Z}_{2}^{5}$ \\
\hline$\{3\}$ & $\left\langle L_{e_{7}+e_{6}}, L_{e_{7}}, L_{e_{5}+e_{6}+e_{3}}, L_{2 e_{j}}: j \neq 1\right\rangle$ & $\mathbb{Z} \oplus \mathbb{Z}_{4} \oplus \mathbb{Z}_{2}^{5}$ \\
\hline$\{4\}$ & $\left\langle L_{e_{7}+e_{6}}, L_{e_{7}+e_{4}}, L_{e_{4}+e_{5}+e_{6}}, L_{2 e_{j}}: j \neq 1\right\rangle$ & $\mathbb{Z} \oplus \mathbb{Z}_{4}^{2} \oplus \mathbb{Z}_{2}^{4}$ \\
\hline$\{2,3\}$ & $\left\langle L_{e_{7}+e_{6}}, L_{e_{7}+e_{2}}, L_{e_{5}+e_{6}+e_{3}}, L_{2 e_{j}}: j \neq 1\right\rangle$ & $\mathbb{Z} \oplus \mathbb{Z}_{4}^{2} \oplus \mathbb{Z}_{2}^{4}$ \\
\hline$\{2,4\}$ & $\left\langle L_{e_{7}+e_{6}}, L_{e_{7}+e_{2}+e_{4}}, L_{e_{5}+}\right.$ & $\mathbb{Z} \oplus \mathbb{Z}_{4}^{2} \oplus \mathbb{Z}_{2}^{4}$ \\
\hline$\{3,4\}$ & $\left\langle L_{e_{7}+e_{6}}, L_{e_{7}+e_{4}}, L_{e_{5}+e_{6}}\right.$ & $\mathbb{Z} \oplus \mathbb{Z}_{4}^{2} \oplus \mathbb{Z}_{2}^{4}$ \\
\hline$\{2,3,4\}$ & $\left\langle L_{e_{7}+e_{6}}, L_{e_{7}+e_{2}+e_{4}}, L_{e_{5}+e}\right.$ & $\mathbb{Z} \oplus \mathbb{Z}_{4}^{2} \oplus \mathbb{Z}_{2}^{4}$ \\
\hline$j=9$ & $\left\langle L_{e_{7}+e_{6}}, L_{e_{7}+e_{2}+e_{4}}, L_{e_{5}+e_{6}}\right.$ & $\mathbb{Z} \oplus \mathbb{Z}_{4}^{3} \oplus \mathbb{Z}_{2}^{3}$ \\
\hline
\end{tabular}

In this case we see that, in addition to the groups obtained in the case $i=1$, we get the group $\mathbb{Z} \oplus \mathbb{Z}_{4}^{3} \oplus \mathbb{Z}_{2}^{3}$. Thus, among the 18 groups obtained, 6 of them have different first integral homology groups. Actually, most of the groups $\Gamma_{i, j}$ should be pairwise non-isomorphic. To check this one should verify whether the cohomology classes in $H^{2}(F, \Lambda)$ corresponding to different $\Gamma_{i, j}$ 's are semilinearly equivalent (see [4], Theorem 2.2).

Example 4.2. We now apply $\mathrm{d}_{q, 0}$ to $\Gamma_{1}, \Gamma_{2}$, two 4-dimensional Bieberbach groups with holonomy group $\mathbb{Z}_{2}$, such that $\Gamma_{i} \backslash \mathbb{R}^{4}$ has a locally invariant Kähler structure, for $i=1,2$. The resulting manifolds will not admit a hyperkähler structure.

Let $\Gamma$ be the Klein-bottle group from Example 4.1. We set

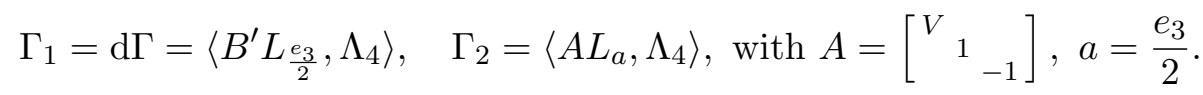

It is clear that $\Gamma_{1}$ is torsion-free and Kähler (see Proposition $3.2(\mathrm{i})$ ) and the same is true of $\Gamma_{2}$ since $(A+I d) a=e_{3} \notin(A+I d) \Lambda_{4}=\mathbb{Z}\left(e_{1}+e_{2}\right) \oplus \mathbb{Z} 2 e_{3}$. To see that $\Gamma_{2}$ is Kähler we note that if $Q=\frac{1}{\sqrt{2}}\left[\begin{array}{ll}1 & 1 \\ 1 & -1\end{array}\right]$ then conjugation of

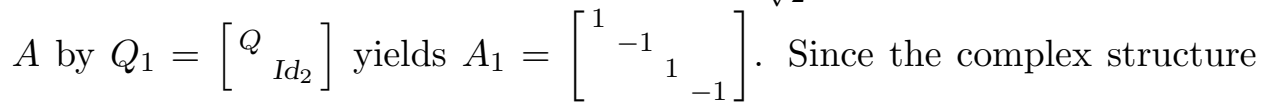
$J_{o}:=\left[\begin{array}{cc}0 & I d_{2} \\ -I d_{2} & 0\end{array}\right]$ commutes with $A_{1}$, then $\tilde{J}:=Q_{1}^{-1} J_{o} Q_{1}$ commutes with $A$. We note that $Q$ is orthogonal.

We now determine all vectors $v \in \mathbb{R}^{4}$ such that $\mathrm{d}_{q, 0}\left(\Gamma_{i}, v\right)$ are Bieberbach groups, $i=1,2$. By Theorem 3.5 (iii), $v=\frac{1}{2} \sum_{1}^{4} e_{i}$ has this property for both $\Gamma_{1}, \Gamma_{2}$. As in Example 4.1 we set $v=\frac{1}{2} \sum_{1}^{4} m_{i} e_{i}$ and look for the values of $m_{i} \in\{0,1\}$ so that (i) in Theorem 3.5 holds. Clearly $\left(B^{\prime}-I d\right) v \in \Lambda_{4}$ for 
any $v$ of this form. However $(A-I d) v \in \Lambda_{4}$ implies that both $m_{1}, m_{2}$ must have the same parity.

The second condition in (i) will be satisfied for $\Gamma_{1}, \Gamma_{2}$, if and only if, respectively,

$$
\begin{gathered}
\left(B^{\prime}+I d\right) v=m_{1} e_{1}+m_{3} e_{3} \notin\left(B^{\prime}+I d\right) \Lambda_{4}=\mathbb{Z} 2 e_{1} \oplus \mathbb{Z} 2 e_{3} \\
(A+I d) v=\frac{m_{1}+m_{2}}{2} e_{1}+\frac{m_{1}+m_{2}}{2} e_{2}+m_{3} e_{3} \notin(A+I d) \Lambda_{4}=\mathbb{Z}\left(e_{1}+e_{2}\right) \oplus \mathbb{Z} 2 e_{3} .
\end{gathered}
$$

This implies that the solutions for $\Gamma_{1}$ are the vectors $v$ such that $m_{1}$ or $m_{3}$ is odd, that is, all vectors of the form $v=\frac{e_{Q}}{2}$, where $e_{Q}=\sum_{j \in Q} e_{j}$ with $Q \subset\{1,2,3,4\}, Q \neq \emptyset,\{2\},\{4\},\{2,4\}$ (a total of 12 solutions).

In the case of $\Gamma_{2}$, the solutions are the vectors such that $m_{1}$ and $m_{2}$ have the same parity and $m_{3}$ is odd, that is, $v=\frac{e_{Q}}{2}$, where $Q$ is one of $\{3\},\{3,4\}$, $\{1,2,3\},\{1,2,3,4\}$ (4 solutions).

For simplicity we shall write $\Gamma_{i, Q}:=\mathrm{d}_{q, 0}\left(\Gamma_{i}, v\right)$ where $i=1,2$ and $Q \subset$ $\{1,2,3,4\}$ is such that $v=\frac{e_{Q}}{2}$ satisfies the conditions above, so that $\Gamma_{i, v}$ is torsion-free. The real cohomology is the same for all manifolds $\mathrm{d}_{q, 0} \Gamma_{i, Q} \backslash \mathbb{R}^{8}$, $i=1,2$, since the holonomy representation over $\mathbb{Q}$ is the same in all cases. The Betti numbers are as usual given by $\beta_{h}=\operatorname{dim} \Lambda^{h} \mathbb{R}^{8^{\mathrm{F}}}, 1 \leq h \leq 8$. Clearly the fixed space for $h=1$ and $h=2$ is generated, respectively, by $\left\{e_{1}, e_{3}\right\}$ and by $\left\{e_{1} \wedge e_{3}, e_{2} \wedge e_{4}, e_{5} \wedge e_{7}\right.$ and $\left.e_{6} \wedge e_{8}\right\}$. For $h=3$, the invariants are obtained by taking exterior products of the form $e_{i} \wedge e_{j} \wedge e_{k}$ where either all three, or one, of $i, j, k$ are $\leq 4$. In the first case we get the vectors $e_{1} \wedge e_{2} \wedge e_{4}$ and $e_{2} \wedge e_{3} \wedge e_{4}$, while in the second there are $2\left(\begin{array}{l}4 \\ 2\end{array}\right)=12$ possibilities, hence $\beta_{3}=14$. Similarly, we compute that $\beta_{4}=1+4^{2}+2^{2}+1=22$. Thus, the Poincaré polynomial of the manifolds $\mathrm{d}_{q, 0} \Gamma_{i, Q} \backslash \mathbb{R}^{8}, i=1,2$ is given by $p(t)=1+2 t+4 t^{2}+14 t^{3}+22 t^{4}+14 t^{5}+4 t^{6}+2 t^{7}+t^{8}$. We note that the Euler characteristic equals $\chi=2-2 \beta_{1}+2 \beta_{2}-2 \beta_{3}+\beta_{4}=2-4+8-28+22=0$, as it should be.

We conclude this example by computing the first integral homology groups of the manifolds $\mathrm{d}_{q, 0} \Gamma_{i, Q} \backslash \mathbb{R}^{8}, i=1,2$.

We have that $\Gamma_{1, v}=\left\langle B^{\prime \prime} L_{\frac{e_{7}}{2}}, E_{4} L_{v}, \Lambda_{8}\right\rangle, \Gamma_{2, v}=\left\langle A^{\prime} L_{\frac{e_{7}}{2}}, E_{4} L_{v}, \Lambda_{8}\right\rangle$, where as usual $A^{\prime}=\left[\begin{array}{ll}A & 0 \\ 0 & A\end{array}\right]$. Furthermore we compute that

$$
\left[B^{\prime \prime} L_{\frac{e_{7}}{2}}, E_{4} L_{v}\right]=L_{e_{7}-p\left(e_{Q}\right)}, \quad\left[A^{\prime} L_{\frac{e_{7}}{2}}, E_{4} L_{v}\right]=L_{e_{7}-p^{\prime}\left(e_{Q}\right)},
$$

where $p, p^{\prime}$ denote the orthogonal projections onto $\mathbb{R} e_{2}+\mathbb{R} e_{4}$ and $\mathbb{R} e_{4}$ respectively, for $i=1,2$. Hence we get that

$$
\begin{gathered}
{\left[\Gamma_{1, v}, \Gamma_{1, v}\right]=\left\langle L_{e_{7}-p\left(e_{Q}\right)}, L_{2 e_{j}}: j \neq 1,3\right\rangle,} \\
{\left[\Gamma_{2, v}, \Gamma_{2, v}\right]=\left\langle L_{e_{7}-p^{\prime}\left(e_{Q}\right)}, L_{2\left(e_{1}-e_{2}\right)}, L_{2 e_{j}}: j \neq 3\right\rangle .}
\end{gathered}
$$

Since $p\left(e_{Q}\right)=0$ (resp. $\left.p^{\prime}\left(e_{Q}\right)=0\right)$ if and only if $Q \subset\{1,3\}$ (resp. $Q=\{3\}$ or $Q=\{1,2,3\})$ and taking into account that $\gamma_{1}^{2}=L_{e_{7}}, \gamma_{3}^{2}=L_{e_{Q}}$ and 
$\left(A L_{\frac{e_{7}}{2}}\right)^{2}=L_{e_{7}}$, we conclude that

$$
\begin{gathered}
\Gamma_{1, v} /\left[\Gamma_{1, v}, \Gamma_{1, v}\right] \simeq \begin{cases}\mathbb{Z}^{2} \oplus \mathbb{Z}_{2}^{6} & \text { if } Q \subset\left\{e_{1}, e_{3}\right\} \\
\mathbb{Z}^{2} \oplus \mathbb{Z}_{4} \oplus \mathbb{Z}_{2}^{5} & \text { if } Q \not \subset\left\{e_{1}, e_{3}\right\}\end{cases} \\
\Gamma_{2, v} /\left[\Gamma_{2, v}, \Gamma_{2, v}\right] \simeq \begin{cases}\mathbb{Z}^{2} \oplus \mathbb{Z}_{2}^{6} & \text { if } 4 \notin Q \\
\mathbb{Z}^{2} \oplus \mathbb{Z}_{4} \oplus \mathbb{Z}_{2}^{5} & \text { if } 4 \in Q .\end{cases}
\end{gathered}
$$

We finally note that, if $\Gamma$ is the Klein bottle group we have 2-fold coverings $M_{\mathrm{d}^{2} \Gamma} \rightarrow M_{\Gamma_{1, Q}}$, where $M_{\mathrm{d}^{2} \Gamma}$ is hyperkähler and $M_{\Gamma_{1, Q}}$ is Kähler and not hyperkähler, since $\beta_{1}\left(M_{\Gamma_{1, Q}}\right)=2$ is not divisible by 4 .

Example 4.3. In the examples discussed so far, the group $\Gamma$ considered lies in the family $\mathcal{F}$. However, examination of particular cases shows that the construction also applies to many groups $\Gamma$ not in $\mathcal{F}$. We shall see in this last example that, for some choice of $\phi$, there exists a vector $v$ so that conditions (i) in Theorem 3.5 are satisfied for all flat manifolds of dimension $n=4$, having holonomy group $\mathbb{Z}_{2}^{2}$, which do not lie in family $\mathcal{F}$. We note, however, that in one case there will be no solution if we choose $\phi=0$.

The groups in dimension $n=4$, which are not in class $\mathcal{F}$ are listed as IV:04/01/04/005, IV:04/01/06/004, V:05/01/06/006, V:05/01/10/004 and $\mathrm{V}: 05 / 01 / 07 / 004$ in [1], but for simplicity, we shall denote them respectively by $\Gamma_{i}, 1 \leq i \leq 5$. We have $\Gamma_{i}=\left\langle A_{i} L_{a_{i}}, B_{i} L_{b_{i}}, \Lambda_{4}\right\rangle$, where $\Lambda_{4}$ is the canonical lattice in $\mathbb{R}_{4}$ and $A_{i}, B_{i}, a_{i}, b_{i}$ are given respectively as follows:

$$
\begin{aligned}
& A_{1}=\left[\begin{array}{cccc}
1 & 0 & 0 & -1 \\
0 & 1 & 0 & 0 \\
0 & 0 & 1 & 1 \\
0 & 0 & 0 & -1
\end{array}\right], \quad a_{1}=\frac{e_{2}}{2}, \quad B_{1}=\left[\begin{array}{cccc}
1 & 0 & 0 & 0 \\
0 & 1 & 0 & 0 \\
0 & 0 & -1 & -1 \\
0 & 0 & 0 & 1
\end{array}\right], \quad b_{1}=\frac{e_{1}}{2} \\
& A_{2}=\left[\begin{array}{cccc}
1 & 0 & 0 & 1 \\
0 & 1 & 0 & 0 \\
0 & 0 & 0 \\
0 & 0 & 0 & -1
\end{array}\right], \quad a_{2}=\frac{e_{3}}{2}, \quad B_{2}=\left[\begin{array}{cccc}
1 & 0 & 1 & 0 \\
0 & 1 & 0 & 0 \\
0 & 0 & -1 & 0 \\
0 & 0 & 0 & 1
\end{array}\right], \quad b_{2}=\frac{e_{2}+e_{4}}{2} \\
& A_{3}=\left[\begin{array}{cccc}
0 & -1 & 1 & 0 \\
-1 & 0 & 1 & 0 \\
0 & 0 & 1 & 0 \\
0 & 0 & 0 & -1
\end{array}\right], a_{3}=\frac{e_{1}+e_{3}+e_{4}}{2}, \quad B_{3}=\left[\begin{array}{cccc}
0 & 1 & -1 & 0 \\
0 & 1 & 0 & 0 \\
-1 & 1 & 0 & 0 \\
0 & 0 & 0 & -1
\end{array}\right], b_{3}=\frac{e_{1}+e_{2}}{2} \\
& A_{4}=\left[\begin{array}{cccc}
0 & 1 & 0 & 0 \\
1 & 0 & 0 & 0 \\
0 & 0 & 1 & 0 \\
0 & 0 & 0 & -1
\end{array}\right], \quad a_{4}=\frac{e_{3}+e_{4}}{2}, \quad B_{4}=\left[\begin{array}{cccc}
1 & 0 & -1 & 0 \\
0 & 1 & -1 & 0 \\
0 & 0 & -1 & 0 \\
0 & 0 & 0 & -1
\end{array}\right], \quad b_{4}=\frac{e_{1}}{2} \\
& A_{5}=\left[\begin{array}{cccc}
1 & 0 & 0 & 1 \\
0 & 1 & 0 & 1 \\
0 & 0 & -1 & 0 \\
0 & 0 & 0 & -1
\end{array}\right], \quad a_{5}=\frac{e_{1}+e_{3}}{2}, \quad B_{5}=\left[\begin{array}{cccc}
1 & 0 & 0 & 0 \\
0 & -1 & 0 & -1 \\
0 & 0 & -1 & -1 \\
0 & 0 & 0 & 1
\end{array}\right], \quad b_{5}=\frac{e_{1}}{2}
\end{aligned}
$$

We shall next show that in all cases except one, if we take $\phi=0$, there exists $v$ satisfying the conditions in Theorem 3.5, (i).

We note that $2 b \in \Lambda$ for any $B L_{b} \in \Gamma_{i}$, in all five cases. Also, (i) requires that $\left(A_{i}-I d\right) v$ and $\left(B_{i}-I d\right) v$ lie in $\Lambda$, for $1 \leq i \leq 5$ and this will impose some restrictions on $v$ in each case. We note that, if $\left(A_{i}-I d\right) v$ and $\left(B_{i}-I d\right) v$ lie in $\Lambda$, then $\left(A_{i} B_{i}-I d\right) v=A_{i}\left(B_{i}-I d\right) v+\left(A_{i}-I d\right) v \in \Lambda$. The second condition in (i) requires that for each $B L_{b} \in \Gamma_{i}(1 \leq i \leq 5)$ we have $(B+I d) v \notin(B+I d) \Lambda$ or $(B-I d) b \notin(B-I d) \Lambda$. We set $v=\frac{1}{2} \sum_{j=1}^{4} m_{j} e_{j}$ with $m_{i} \in \mathbb{Z}$ and at least one of the $m_{i}$ 's odd. We note that, since only the class of $v$ modulo $\Lambda$ matters, we may assume that $m_{j}=0,1$ for each $i$. For each $\Gamma_{i}, 1 \leq i \leq 5$ one computes: 
Case of $\Gamma_{1}$ :

$$
\begin{aligned}
& \left(A_{1}-I d\right) v=\frac{1}{2}\left(-m_{4}, 0, m_{4},-2 m_{4}\right) \\
& \left(B_{1}-I d\right) v=\frac{1}{2}\left(0,0,-2 m_{3}-m_{4}, 0\right) \\
& \left(A_{1}+I d\right) v=\frac{1}{2}\left(2 m_{1}-m_{4}, 2 m_{2}, 2 m_{3}+m_{4}, 0\right) \\
& \left(A_{1}+I d\right) \Lambda=\left\{\left(2 n_{1}-n_{4}, 2 n_{2}, 2 n_{3}+n_{4}, 0\right): n_{j} \in \mathbb{Z}\right\} \\
& \left(B_{1}+I d\right) v=\frac{1}{2}\left(2 m_{1}, 2 m_{2},-m_{4}, 2 m_{4}\right) \\
& \left(B_{1}+I d\right) \Lambda=\left\{\left(2 n_{1}, 2 n_{2},-n_{4}, 2 n_{4}\right): n_{j} \in \mathbb{Z}\right\} \\
& \left(A_{1} B_{1}+I d\right) v=\frac{1}{2}\left(2 m_{1}-m_{4}, 2 m_{2}, m_{4}, 0\right) \\
& \left(A_{1} B_{1}+I d\right) \Lambda=\left\{\left(2 n_{1}-n_{4}, 2 n_{2}, n_{4}, 0\right): n_{j} \in \mathbb{Z}\right\}
\end{aligned}
$$

We see that the first equation implies that $m_{4}$ must be even. If furthermore $m_{2}$ is odd, all conditions in (i) of Theorem 3.5 hold. For instance $v=\frac{e_{2}}{2}$ is a solution.

Case of $\Gamma_{2}$ :

$$
\begin{aligned}
& \left(A_{2}-I d\right) v=\frac{1}{2}\left(m_{4}, 0,0,-2 m_{4}\right) \\
& \left(B_{2}-I d\right) v=\frac{1}{2}\left(m_{3}, 0,-2 m_{3}, 0\right) \\
& \left(A_{2}+I d\right) v=\frac{1}{2}\left(2 m_{1}+m_{4}, 2 m_{2}, 2 m_{3}, 0\right) \\
& \left(A_{2}+I d\right) \Lambda=\left\{\left(2 n_{1}+n_{4}, 2 n_{2}, 2 n_{3}, 0\right): n_{j} \in \mathbb{Z}\right\} \\
& \left(B_{2}+I d\right) v=\frac{1}{2}\left(2 m_{1}+m_{3}, 2 m_{2}, 0,2 m_{4}\right) \\
& \left(B_{2}+I d\right) \Lambda=\left\{\left(2 n_{1}+n_{3}, 2 n_{2}, 0,2 n_{4}\right): n_{j} \in \mathbb{Z}\right\} \\
& \left(A_{2} B_{2}+I d\right) v=\frac{1}{2}\left(2 m_{1}+m_{3}+m_{4}, 2 m_{2}, 0,0\right) \\
& \left(A_{2} B_{2}+I d\right) \Lambda=\left\{\left(2 n_{1}+n_{3}+n_{4}, 2 n_{2}, 0,0\right): n_{j} \in \mathbb{Z}\right\}
\end{aligned}
$$

We see that the first and second equations imply that $m_{3}$ and $m_{4}$ must be even and the second condition in (i) of Theorem 3.5 forces $m_{2}$ to be odd. These are the only conditions on $v$. The vector $v=\frac{e_{2}}{2}$ is a solution.

Case of $\Gamma_{3}$ :

$$
\begin{aligned}
& \left(A_{3}-I d\right) v=\frac{1}{2}\left(-m_{1}-m_{2}+m_{3},-m_{1}-m_{2}+m_{3}, 0,-2 m_{4}\right) \\
& \left(B_{3}-I d\right) v=\frac{1}{2}\left(-m_{1}+m_{2}-m_{3}, 0,-m_{1}+m_{2}-m_{3},-2 m_{4}\right) \\
& \left(A_{3}+I d\right) v=\frac{1}{2}\left(m_{1}-m_{2}+m_{3},-m_{1}+m_{2}+m_{3}, 2 m_{3}, 0\right) \\
& \left(A_{3}+I d\right) \Lambda=\left\{\left(n_{1}-n_{2}+n_{3},-n_{1}+n_{2}+n_{3}, 2 n_{3}, 0\right): n_{j} \in \mathbb{Z}\right\} \\
& \left(B_{3}+I d\right) v=\frac{1}{2}\left(m_{1}+m_{2}-m_{3}, 2 m_{2},-m_{1}+m_{2}+m_{3}, 0\right) \\
& \left(B_{3}+I d\right) \Lambda=\left\{\left(n_{1}+n_{2}-n_{3}, 2 n_{2},-n_{1}+n_{2}+n_{3}, 0\right): n_{j} \in \mathbb{Z}\right\} \\
& \left(A_{3} B_{3}+I d\right) v=\frac{1}{2}\left(0,-m_{1}+m_{2}+m_{3},-m_{1}+m_{2}+m_{3}, 2 m_{4}\right) \\
& \left(A_{3} B_{3}+I d\right) \Lambda=\left\{\left(0,-n_{1}+n_{2}+n_{3},-n_{1}+n_{2}+n_{3}, 2 n_{4}\right): n_{j} \in \mathbb{Z}\right\}
\end{aligned}
$$

Now the first two equations imply that $m_{1}+m_{2}+m_{3}$ is even and we easily see that if we take $v=\frac{e_{2}+e_{3}+e_{4}}{2}$, all conditions in Theorem 3.5 are satisfied.

Case of $\Gamma_{4}$ :

$$
\begin{aligned}
& \left(A_{4}-I d\right) v=\frac{1}{2}\left(m_{2}-m_{1}, m_{1}-m_{2}, 0,-2 m_{4}\right) \\
& \left(B_{4}-I d\right) v=\frac{1}{2}\left(-m_{3},-m_{3},-2 m_{3},-2 m_{4}\right) \\
& \left(A_{4}+I d\right) v=\frac{1}{2}\left(m_{1}+m_{2}, m_{1}+m_{2}, 2 m_{3}, 0\right) \\
& \left(A_{4}+I d\right) \Lambda=\left\{\left(n_{1}+n_{2}, n_{1}+n_{2}, 2 n_{3}, 0\right): n_{j} \in \mathbb{Z}\right\}
\end{aligned}
$$




$$
\begin{aligned}
& \left(B_{4}+I d\right) v=\frac{1}{2}\left(2 m_{1}-m_{3}, 2 m_{2}-m_{3}, 0,0\right) \\
& \left(B_{4}+I d\right) \Lambda=\left\{\left(2 n_{1}-n_{3}, 2 n_{2}-n_{3}, 0,0\right): n_{j} \in \mathbb{Z}\right\} \\
& \left(A_{4} B_{4}+I d\right) v=\frac{1}{2}\left(2 m_{1}-m_{4}, 2 m_{2}, 2 m_{3}+m_{4}, 0\right) \\
& \left(A_{4} B_{4}+I d\right) \Lambda=\left\{\left(2 n_{1}-n_{4}, 2 n_{2}, n_{4}, 0\right): n_{j} \in \mathbb{Z}\right\} \\
& \left(A_{4}-I d\right) a_{4}=(0,0,0,-1) \notin\left(A_{4}-I d\right) \Lambda, \quad\left(B_{4}-I d\right) b_{4}=0 .
\end{aligned}
$$

We see in this case that the first two equations impose many restrictions, namely, $m_{1}, m_{2}$ must have the same parity and $m_{3}$ must be even. On the other hand, one immediately sees that for any $v$ which satisfies these restrictions, one has that $\left(B_{4}+I d\right) v \in\left(B_{4}+I d\right) \Lambda$. Hence there is no $v$ in this case which verifies all conditions. (We note that, since $\left(A_{4}-I d\right) a_{4} \notin\left(A_{4}-I d\right) \Lambda$, we need not check the condition on $\left(A_{4}+I d\right) v$.) However, we shall see that if we form $\mathrm{d}_{\phi} \Gamma$, with $\phi$ the 1-cocycle associated to $\Gamma$ (see Remark 3.3), then there is a solution. We have:

$\left(A_{4}+I d\right)\left(v+a_{4}\right)=\frac{1}{2}\left(m_{1}+m_{2}, m_{1}+m_{2}, 2 m_{3}+2,0\right) \notin\left(A_{4}+I d\right) \Lambda=$ $\left\{\left(n_{1}+n_{2}, n_{1}+n_{2}, 2 n_{3}, 0\right): n_{j} \in \mathbb{Z}\right\}$, since $m_{3}$ is even.

$\left(B_{4}+I d\right)\left(v+b_{4}\right)=\frac{1}{2}\left(2 m_{1}-m_{3}+2,2 m_{2}-m_{3}, 0,0\right) \notin\left(B_{4}+I d\right) \Lambda=$ $\left\{\left(2 n_{1}-n_{3}, 2 n_{2}-n_{3}, 0,0\right): n_{j} \in \mathbb{Z}\right\}$ for any choice of $v$, since $m_{1}$ and $m_{2}$ have the same parity.

$\left(A_{4} B_{4}+I d\right)\left(v+b_{A_{4} B_{4}}\right)=\frac{1}{2}\left(m_{1}+m_{2}+m_{3}, m_{1}+m_{2}+m_{3}, 0,2 m_{4}+2\right) \notin$ $\left(A_{4} B_{4}+I d\right) \Lambda=\left\{\left(n_{1}+n_{2}-n_{3}, n_{1}+n_{2}-n_{3}, 0,2 n_{4}\right): n_{j} \in \mathbb{Z}\right\}$, if we choose $v=\frac{e_{1}+e_{2}}{2}$ (here $\left.b_{A_{4} B_{4}}=B_{4} a_{4}+b_{4}\right)$.

We note that $v=\frac{e_{1}+e_{2}}{2}$ is the only solution to all equations.

Case of $\Gamma_{5}$ :

$$
\begin{aligned}
& \left(A_{5}-I d\right) v=\frac{1}{2}\left(m_{4}, m_{4},-2 m_{3},-2 m_{4}\right) \\
& \left(B_{5}-I d\right) v=\frac{1}{2}\left(0,-2 m_{2}-m_{4},-2 m_{3}-m_{4}, 2 m_{4}\right) \\
& \left(A_{5}+I d\right) v=\frac{1}{2}\left(2 m_{1}+m_{4}, 2 m_{2}+m_{4}, 0,0\right) \\
& \left(A_{5}+I d\right) \Lambda=\left\{\left(2 n_{1}+n_{4}, 2 n_{2}+n_{4}, 0,0\right): n_{j} \in \mathbb{Z}\right\} \\
& \left(B_{5}+I d\right) v=\frac{1}{2}\left(2 m_{1},-m_{4},-m_{4}, 2 m_{4}\right) \\
& \left(B_{5}+I d\right) \Lambda=\left\{\left(2 n_{1},-n_{4},-n_{4}, 2 n_{4}\right): n_{j} \in \mathbb{Z}\right\} \\
& \left(A_{5} B_{5}+I d\right) v=\frac{1}{2}\left(2 m_{1}+m_{4}, 0,2 m_{3}+m_{4}, 0\right) \\
& \left(A_{5} B_{5}+I d\right) \Lambda=\left\{\left(2 n_{1}+n_{4}, 0,2 n_{3}+n_{4}, 0\right): n_{j} \in \mathbb{Z}\right\}
\end{aligned}
$$

We see that the first equation implies that $m_{4}$ is even. We may take $m_{1}=1$ and $m_{2}=0$ and all conditions in (i) of Theorem 3.5 are satisfied. Hence $v=\frac{e_{1}}{2}$ is a solution. We note in particular that $\left(A_{5} B_{5}+I d\right) \frac{e_{1}}{2}=e_{1} \notin$ $\left(A_{5} B_{5}+I d\right) \Lambda$, since $2 n_{1}+n_{4}$ and $2 n_{3}+n_{4}$ always have the same parity.

\section{REFERENCES}

[1] Brown H., Bülow R., Neubüser J., Wondracek H., Zassenhaus H. Crystallographic groups of dimension four, John Wiley, 1978.

[2] Benson Ch., Gordon C., Kähler and symplectic structures on nilmanifolds, Topology 27, 1988, 513-518.

[3] Barberis M.L., Dotti I.G., Miatello R.J., Clifford structures on certain locally homogeneous manifolds, Annals Global Analysis and Geometry 13, 1995, 289-301.

[4] Charlap. L., Bieberbach groups and flat manifolds, Springer Verlag, Universitext, 1988. 
[5] Cobb P., Manifolds with holonomy group $\mathbb{Z}_{2} \oplus \mathbb{Z}_{2}$ and first Betti number zero, Jour. Differential Geometry 10, 1975, 221-224.

[6] Dotti I.G., Miatello R.J., Isospectral compact flat manifolds, Duke Math. Jour. 68, 1992, 489-498.

[7] Hiller H., Cohomology of Bieberbach groups, Mathematika 32, 1986, 55-59.

[8] Miatello R.J., Rossetti J.P., Isospectral Hantzsche-Wendt manifolds, Journal für die Reine und Angewandte Mathematik 515, 1999.

[9] Miatello R.J., Rossetti J.P., Flat manifolds isospectral on p-forms, preprint,1999.

[10] Nazarova L.A., Unimodular representations of the 4-group, Soviet Math. Dokl 2, 11961, 1304-1307.

[11] Rossetti J.P., Tirao P.A., Compact Flat manifolds with holonomy group $\mathbb{Z}_{2} \oplus \mathbb{Z}_{2}$, Proc. A.M.S. 124, 1996, 2491-2499.

[12] Rossetti J.P., Tirao P.A., Five-dimensional Bieberbach groups with holonomy group $\mathbb{Z}_{2} \oplus \mathbb{Z}_{2}$, Geometriae Dedicata 77, 1999, 149-172.

[13] Rossetti J.P., Tirao P.A., Compact Flat manifolds with holonomy group $\mathbb{Z}_{2} \oplus \mathbb{Z}_{2}$ II, Rendic. Sem. Matem. Univ. di Padova 101, 1999, 99-136.

[14] Wolf J.A., Spaces of constant curvature, Mc Graw-Hill, NY, 1967.

Facultad de Matemática, Astronomía y Física, Universidad Nacional de Córdoba, Córdoba 5000, Argentina

E-mail address: idotti@mate.uncor.edu, miatello@mate.uncor.edu 